\title{
gु \\ Electron-phonon coupling in graphene antidot lattices: An indication of polaronic behavior
}

\author{
Nenad Vukmirović, ${ }^{1}$ Vladimir M. Stojanović, ${ }^{2, *}$ and Mihajlo Vanević3 ${ }^{3,4}$ \\ ${ }^{1}$ Computational Research Division, Lawrence Berkeley National Laboratory, Berkeley, California 94720, USA \\ ${ }^{2}$ Department of Physics, University of Basel, Klingelbergstrasse 82, CH-4056 Basel, Switzerland \\ ${ }^{3}$ School of Physics, Georgia Institute of Technology, Atlanta, Georgia 30332, USA \\ ${ }^{4}$ Kavli Institute of Nanoscience, Delft University of Technology, 2628 CJ Delft, The Netherlands
}

(Received 18 September 2009; published 8 January 2010)

\begin{abstract}
We study graphene antidot lattices — superlattices of perforations (antidots) in a graphene sheet-using a model that accounts for the phonon modulation of the $\pi$-electron hopping integrals. We calculate the phonon spectra of selected antidot lattices using two different semiempirical methods. Based on the adopted model, we quantify the nature of charge carriers in the system by computing the quasiparticle weight due to the electronphonon interaction for an excess electron in the conduction band. We find a very strong phonon-induced renormalization, with the effective electron masses exhibiting nonmonotonic dependence on the superlattice period for a given antidot diameter. Our study provides an indication of polaronic behavior and points to the necessity of taking into account the inelastic degrees of freedom in future studies of transport in graphene antidot lattices.
\end{abstract}

DOI: 10.1103/PhysRevB.81.041408

PACS number(s): 71.38.-k, 63.20.kd, 63.22.- m, 73.21.Cd

Recent years have seen a surge of interest in graphenethe two-dimensional form of carbon with atoms ordered in a honeycomb lattice. ${ }^{1}$ This material shows extraordinary properties, such as room-temperature ballistic transport on a submicron scale and the possibility of heavy doping without altering significantly the charge-carrier mobility. Yet, the usefulness of pure graphene for carbon-based electronics ${ }^{2}$ is limited as the electron transmission probability across a potential barrier is always unity-regardless of the height and width of the barrier-a feature akin to Klein tunneling. ${ }^{3}$ Thus the conductivity cannot be altered by a gate voltage, the latter being a key property of a field-effect transistor.

Motivated in part by the compelling need to create a band gap in graphene, an extensive research effort is currently being dedicated to understanding the electronic properties of graphene-based superlattices. ${ }^{4}$ A class of such structures, made by perforating a graphene sheet-graphene antidot lattices-has recently been proposed. ${ }^{5}$ These lattices belong to the family of superhoneycomb systems ${ }^{6}$ and can be obtained by patterning graphene monolayers using electronbeam lithography, a method which allows feature sizes as small as tens of nanometers. It is worthwhile to stress, however, that-owing to recent advances in nanofabrication ${ }^{7}$ - sub-10 $\mathrm{nm}$ antidot diameters constitute a realistic near-future prospect.

The electronic structure of triangular antidot lattices has been studied theoretically, ${ }^{8,9}$ revealing features such as the existence of localized midgap states (flat- and quasiflat bands). In addition, transport properties of their squarelattice counterparts have been investigated experimentally, ${ }^{10}$ showing a transport gap and weak localization corrections to the conductance.

In the present work, we study the influence of phonons on the electronic properties of graphene antidot lattices. We calculate the phonon spectra of selected antidot lattices using two independent semiempirical methods. We then describe the electron-phonon (henceforth e-ph) interaction based on a model that accounts for the modulation of hopping integrals by the lattice displacements (Peierls-type e-ph coupling). ${ }^{11}$ Within this model, we quantify the effect of phonons by computing the conduction-band quasiparticle spectral weight. We show that the phonon-induced renormalization is much stronger than in graphene itself, with the effective electron masses being typically in the range $3.7-5$ bare band masses.

The triangular graphene antidot lattice $\{L, R\}$ with circular perforations [see Fig. 1(a)] has a hexagonal unit cell [Fig. 1(b)] with side length $L a$ and antidot radius $R a$, where $a=2.46 \AA$ is the lattice constant of graphene. If we choose a carbon atom (hereafter $\mathrm{C}$ atom) on sublattice $\mathrm{A}$ as the origin, its nearest neighbors are given by the vectors $\boldsymbol{\delta}_{1}=(\sqrt{3} / 2,-1 / 2) a_{c c}, \quad \boldsymbol{\delta}_{2}=(0,1) a_{c c}, \quad$ and $\boldsymbol{\delta}_{3}=(-\sqrt{3} / 2,-1 / 2) a_{c c}$ [Fig. 1(b)], with $a_{c c}=1.42 \AA$ being the distance between adjacent $\mathrm{C}$ atoms.

Given the large size of unit cells in the antidot lattices that we consider-with $N_{\text {at }} \sim 300-1600$ atoms - the calculation of the electronic structure and the phonon spectra in the whole Brillouin zone using $a b$ initio methods based on the density functional theory (DFT) is inconceivable. Instead, we model the band structure using a nearest-neighbor tightbinding Hamiltonian ${ }^{8}$

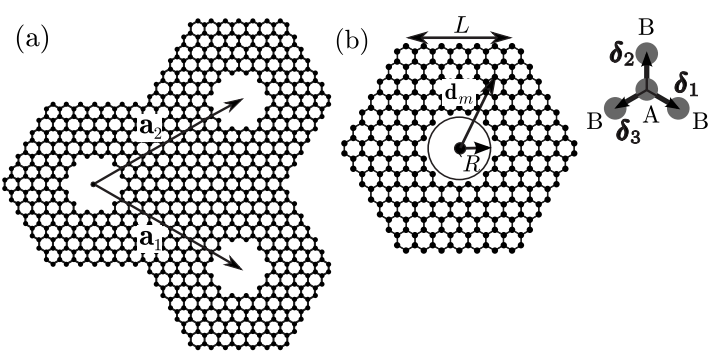

FIG. 1. (a) A segment of a triangular graphene antidot lattice with circular antidots and basis vectors $\mathbf{a}_{1}$ and $\mathbf{a}_{2}$. The lattice period is $\left|\mathbf{a}_{1}\right|=\left|\mathbf{a}_{2}\right|=L a \sqrt{3}$. (b) unit cell of an antidot lattice, with vectors $\boldsymbol{\delta}_{1}, \boldsymbol{\delta}_{2}$, and $\boldsymbol{\delta}_{3}$ specifying positions of the nearest neighbors of a carbon atom on sublattice $A$. 


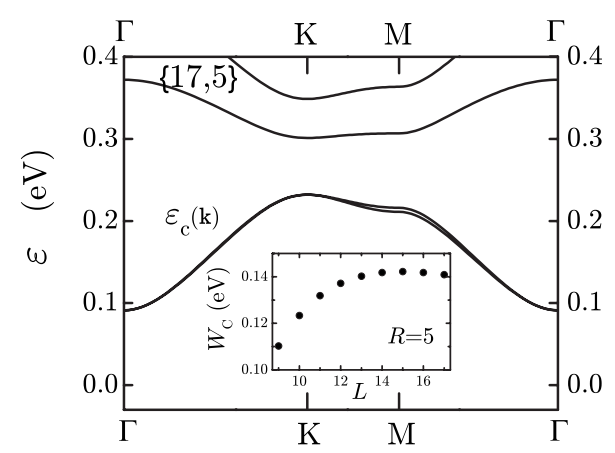

FIG. 2. The conduction-band dispersion $\varepsilon_{c}(\mathbf{k})$ for the $\{17,5\}$ antidot lattice. The inset shows the $L$-dependence $(9 \leq L \leq 17)$ of the conduction bandwidth $W_{c}$ for $R=5$.

$$
\hat{H}_{\mathrm{e}}=-\frac{t}{2} \sum_{\mathbf{R}, m, \boldsymbol{\delta}}\left(\hat{a}_{\mathbf{R}+\mathbf{d}_{m}+\boldsymbol{\delta}}^{\dagger} \hat{\boldsymbol{d}}_{\mathbf{R}+\mathbf{d}_{m}}+\text { H.c. }\right),
$$

where $\mathbf{R}$ designate the unit cells ( $N$ of them), $\mathbf{d}_{m}\left(m=1, \ldots, N_{\mathrm{at}}\right)$ specify the positions of the $\mathrm{C}$ atoms within a unit cell, $\boldsymbol{\delta}$ stands for the nearest neighbors of the $\mathrm{C}$ atom at position $\mathbf{R}+\mathbf{d}_{m}$, and $t \approx 2.8 \mathrm{eV}$ is the nearest-neighbor hopping integral. Within our model, the Bloch wave functions corresponding to the energy eigenvalues $\varepsilon_{n}(\mathbf{k})$ ( $n$ is the band index) are given by $\psi_{n \mathbf{k}}(\mathbf{r})=\Sigma_{m} C_{m}^{n, \mathbf{k}} \phi_{m \mathbf{k}}(\mathbf{r})$, where $\phi_{m \mathbf{k}}(\mathbf{r})=N^{-1 / 2} \Sigma_{\mathbf{R}} e^{i \mathbf{k} \cdot \mathbf{R}} \varphi\left(\mathbf{r}-\mathbf{R}-\mathbf{d}_{m}\right)$ and $\varphi\left(\mathbf{r}-\mathbf{R}-\mathbf{d}_{m}\right)$ is the $2 p_{z}$ orbital of a $\mathbf{C}$ atom at $\mathbf{R}+\mathbf{d}_{m}$. To a good approximation, the overlap of the $2 p_{z}$ orbitals on different atoms can be neglected.

The accuracy of the tight-binding method in the case of antidot lattices 8 is corroborated by the recently demonstrated good agreement with the DFT results for lattices with very small unit cells. ${ }^{9}$ Given that the underlying honeycomb lattice is bipartite, the resulting tight-binding energy spectrum has particle-hole symmetry ${ }^{8}$ - a property not retained in the exact band structure. ${ }^{9}$ Our calculations show (see Fig. 2 for an illustration) that the antidot lattices are extremely narrow-band systems: for instance, in the $\{L, 5\}$ family (with $9 \leq L \leq 17$ ) of lattices the conduction bandwidth $W_{c}$ increases from 0.11 to $0.14 \mathrm{eV}$ (see the inset of Fig. 2); in the $\{L, 7\}$ family $(12 \leq L \leq 17)$ it increases from 0.020 to 0.035 $\mathrm{eV}$. The band gap decreases from 0.74 to $0.18 \mathrm{eV}$ in the $\{L, 5\}$ and from 0.30 to $0.15 \mathrm{eV}$ in the $\{L, 7\}$ family.

The phonon spectrum of graphene was studied extensively, using either $a b$ initio methods or effective models. ${ }^{12}$ In the present work, we calculate the phonon spectra of graphene antidot lattices using two independent methods that have recently been shown to yield very accurate results for graphene: the fourth-nearest-neighbor force-constant (4NNFC) method, in the parametrization of Zimmermann et $a l$, and the valence force field (VFF) method of Perebeinos and Tersoff. ${ }^{12}$

We study the phonon spectra for the $\{L, 5\}$ and $\{L, 7\}$ antidot lattices. In each case, we first find the equilibrium lattice configuration by relaxing the atoms until forces on them are smaller than $10^{-5} \mathrm{eV} / \AA$. We then construct the force-constant matrix $D_{m \beta, m^{\prime} \beta^{\prime}}\left(\mathbf{R}-\mathbf{R}^{\prime}\right)$ $\equiv \partial^{2} E_{\text {tot }} / \partial u_{m \beta}(\mathbf{R}) \partial u_{m^{\prime} \beta^{\prime}}\left(\mathbf{R}^{\prime}\right)$, where $u_{m \beta}(\mathbf{R})$ are the displace-

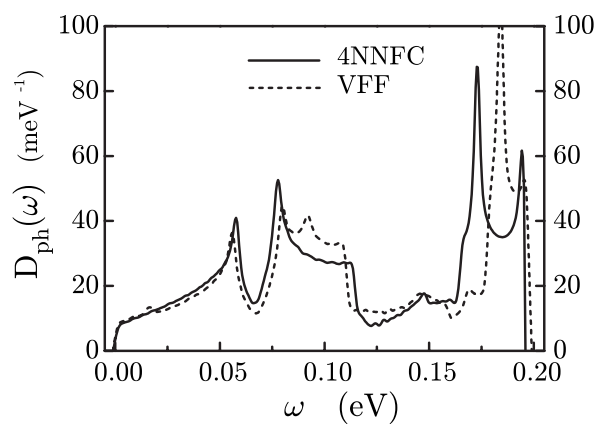

FIG. 3. The phonon density of states for the $\{17,5\}$ antidot lattice, obtained using the 4NNFC (solid line) and VFF (dashed line) methods.

ments $(\beta=x, y, z)$ from the equilibrium position for an atom at $\mathbf{R}+\mathbf{d}_{m}$, and $E_{\mathrm{tot}}$ the total lattice potential energy. The normal-mode frequencies $\omega_{\lambda}(\mathbf{q})$ and eigenvectors $\mathbf{v}^{\lambda}(\mathbf{q})(\lambda$ is the phonon branch index) are obtained from the eigenvalue problem $\mathbf{D}(\mathbf{q}) \mathbf{v}^{\lambda}(\mathbf{q})=M \omega_{\lambda}^{2}(\mathbf{q}) \mathbf{v}^{\lambda}(\mathbf{q})$ for the dynamical matrix $\mathbf{D}(\mathbf{q}) \equiv \Sigma_{\mathbf{R}} \mathbf{D}(\mathbf{R}) e^{-i \mathbf{q} \cdot \mathbf{R}}$, with $M$ being the C-atom mass.

The salient feature of the obtained phonon spectra is that the highest optical-phonon energy at $\mathbf{q}=0$ is at around 195.3 meV-essentially inherited from graphene itself and only weakly dependent on $L$ and $R$-while the lowest optical-phonon energy can be as low as $0.69 \mathrm{meV}$, the case of the $\{17,5\}$ antidot lattice. The two methods used are compared by computing the phonon density of states $D_{\mathrm{ph}}(\omega) \equiv N^{-1} \Sigma_{\mathbf{q}, \lambda} \delta\left[\omega-\omega_{\lambda}(\mathbf{q})\right]$, which shows their good agreement (Fig. 3), especially in the low-energy part of the phonon spectrum.

Generally speaking, the dominant mechanism of the e-ph coupling in the $\pi$-electron systems is the phonon modulation of the electronic hopping integrals ${ }^{13-15}$-Peierls-type coupling. ${ }^{11}$ The latter forms the basis of the Su-Schrieffer-Heeger (SSH) model. ${ }^{13,16}$ We thus adopt a model comprising an electron term $\left(\hat{H}_{\mathrm{e}}\right)$, the phonon term $(\hbar=1) \quad \hat{H}_{\mathrm{ph}}=\Sigma_{\mathbf{q}, \lambda} \omega_{\lambda}(\mathbf{q})\left(\hat{b}_{\mathbf{q}, \lambda}^{\dagger} \hat{b}_{\mathbf{q}, \lambda}+1 / 2\right)$, and a Peierls-type e-ph coupling term

$\hat{H}_{\mathrm{ep}}=\frac{\alpha}{2} \sum_{\mathbf{R}, m, \boldsymbol{\delta}, \lambda}\left(\hat{a}_{\mathbf{R}+\mathbf{d}_{m}+\boldsymbol{\delta}}^{\dagger} \hat{a}_{\mathbf{R}+\mathbf{d}_{m}}+\right.$ H.c. $)\left[\hat{\mathbf{u}}_{\lambda, \mathbf{R}+\mathbf{d}_{m}+\boldsymbol{\delta}}-\hat{\mathbf{u}}_{\lambda, \mathbf{R}+\mathbf{d}_{m}}\right] \cdot \overline{\boldsymbol{\delta}}$,

where $\overline{\boldsymbol{\delta}} \equiv \boldsymbol{\delta} /\|\boldsymbol{\delta}\|$ is the unit vector in the direction of $\boldsymbol{\delta}$,

$$
\hat{\mathbf{u}}_{\lambda, \mathbf{R}+\mathbf{d}_{m}} \equiv \frac{1}{\sqrt{N}} \sum_{\mathbf{q}} \frac{e^{i \mathbf{q} \cdot \mathbf{R}}\left(\hat{b}_{-\mathbf{q}, \lambda}^{\dagger}+\hat{b}_{\mathbf{q}, \lambda}\right)}{\sqrt{2 M \omega_{\lambda}(\mathbf{q})}} \mathbf{v}_{m}^{\lambda}(\mathbf{q})
$$

is the displacement of an atom at position $\mathbf{R}+\mathbf{d}_{m}$ due to the (optical) phonon branch $\lambda$, and $\alpha=5.27 \mathrm{eV} / \AA$ the e-ph coupling constant describing the linear dependence of the $\pi$-electron hopping integrals upon the $\mathrm{C}$-C bond-length modulations. ${ }^{14}$ In the last equation, the phonon eigenvectors $\mathbf{v}_{m}^{\lambda}(\mathbf{q})$ are normalized such that $\Sigma_{m} \mathbf{v}_{m}^{\lambda}(\mathbf{q}) \cdot\left[\mathbf{v}_{m}^{\lambda^{\prime}}(\mathbf{q})\right]^{*}=N_{\text {at }} \delta_{\lambda \lambda^{\prime}}$ and $\mathbf{v}_{m}^{\lambda}(-\mathbf{q})=\left[\mathbf{v}_{m}^{\lambda}(\mathbf{q})\right]^{*} \cdot{ }^{17}$

In momentum space, the Hamiltonian of Eq. (2) reads as 


$$
\hat{H}_{\mathrm{ep}}=\frac{1}{\sqrt{N}} \sum_{\mathbf{k}, \mathbf{q}, \lambda, n} \gamma_{n n}^{\lambda}(\mathbf{k}, \mathbf{q}) \hat{a}_{n, \mathbf{k}+\mathbf{q}}^{\dagger} \hat{a}_{n, \mathbf{k}}\left(\hat{b}_{-\mathbf{q}, \lambda}^{\dagger}+\hat{b}_{\mathbf{q}, \lambda}\right),
$$

where $\hat{a}_{n, \mathbf{k}}^{\dagger}$ creates an electron in a Bloch state $\psi_{n \mathbf{k}}$ (eigenstate of $\left.\hat{H}_{\mathrm{e}}\right)$ and $\gamma_{n n}^{\lambda}(\mathbf{k}, \mathbf{q})$ stands for the e-ph interaction vertex function. It can be shown that the latter is given by $\gamma_{n n}^{\lambda}(\mathbf{k}, \mathbf{q})=V_{n n}^{\lambda}(\mathbf{k}, \mathbf{q})+W_{n n}^{\lambda}(\mathbf{k}, \mathbf{q})$, where

$$
\begin{aligned}
V_{n n}^{\lambda}(\mathbf{k}, \mathbf{q})= & \frac{\alpha}{\sqrt{8 M \omega_{\lambda}(\mathbf{q})}} \sum_{m, \boldsymbol{\delta}} \overline{\boldsymbol{\delta}} \cdot\left[\mathbf{v}_{m+\delta}^{\lambda}(\mathbf{q})-\mathbf{v}_{m}^{\lambda}(\mathbf{q})\right] \\
& \times\left[\left(C_{m+\delta}^{n, \mathbf{k}+\mathbf{q}}\right)^{*} C_{m}^{n, \mathbf{k}}+\left(C_{m}^{n, \mathbf{k}+\mathbf{q}}\right)^{*} C_{m+\delta}^{n, \mathbf{k}}\right]
\end{aligned}
$$

is the contribution due to hopping within a single unit cell (the indices $m+\delta$ denote neighbors $\mathbf{d}_{m}+\boldsymbol{\delta}$ of site $\mathbf{d}_{m}$ and the coefficients $C_{m}^{n, \mathbf{k}}$ originate from the aforementioned tightbinding band structure calculation), while

$$
\begin{aligned}
W_{n n}^{\lambda}(\mathbf{k}, \mathbf{q})= & \frac{\alpha}{\sqrt{8 M \omega_{\lambda}(\mathbf{q})}} \sum_{m, \boldsymbol{\delta}, \mathbf{a}}^{\prime} \overline{\boldsymbol{\delta}} \cdot\left[e^{i \mathbf{q} \cdot \mathbf{a}} \mathbf{v}_{m_{1}}^{\lambda}(\mathbf{q})-\mathbf{v}_{m}^{\lambda}(\mathbf{q})\right] \\
& \times\left[e^{-i(\mathbf{k}+\mathbf{q}) \cdot \mathbf{a}}\left(C_{m_{1}}^{n, \mathbf{k}+\mathbf{q}}\right)^{*} C_{m}^{n, \mathbf{k}}+e^{i \mathbf{k} \cdot \mathbf{a}}\left(C_{m}^{n, \mathbf{k}+\mathbf{q}}\right)^{*} C_{m_{1}}^{n, \mathbf{k}}\right]
\end{aligned}
$$

originates from the hopping between adjacent unit cells. The prime in the last sum signifies a summation restricted to the neighbors $\mathbf{d}_{m}+\boldsymbol{\delta}$ of site $\mathbf{d}_{m}$ that satisfy the condition $\mathbf{d}_{m}+\boldsymbol{\delta}=\mathbf{a}+\mathbf{d}_{m_{1}}$ for some $m_{1}=m_{1}(\boldsymbol{\delta})$, with $\mathbf{a}= \pm \mathbf{a}_{1}, \pm \mathbf{a}_{2}$, $\pm\left(\mathbf{a}_{1}-\mathbf{a}_{2}\right)$. Unlike the more conventional Holstein-type e-ph coupling, which is completely momentum independent, the Peierls-type coupling depends on both the electron and phonon momenta. The momentum dependence of the vertex function is more complicated than that of the standard SSH coupling: while the latter is defined on a monoatomic lattice, here we study a lattice with a basis $\left\{\mathbf{d}_{m}\right\}$. It is straightforward to check, however, that for the case of a monoatomic lattice $\left(N_{\mathrm{at}} \rightarrow 1, C_{m}^{n, \mathbf{k}} \rightarrow \delta_{n m}\right)$ the vertex function takes on the standard SSH dependence $\gamma(\mathbf{k}, \mathbf{q}) \propto[\sin (\mathbf{k} \cdot \mathbf{a})-\sin ((\mathbf{k}+\mathbf{q}) \cdot \mathbf{a})]{ }^{13}$

The overlap of the bare-electron Bloch state $\hat{a}_{n \mathbf{k}}^{\dagger}|0\rangle$ and the Bloch state $\left|\Psi_{n \mathbf{k}}\right\rangle$ of the coupled e-ph system defines the quasiparticle spectral weight $Z_{n}(\mathbf{k}) \equiv\left|\left\langle\Psi_{n \mathbf{k}}\left|\hat{a}_{n \mathbf{k}}^{\dagger}\right| 0\right\rangle\right|^{2}$, a quantity characterizing the renormalization of the electron Green's function by the e-ph interaction. Its inverse is given by ${ }^{18} Z_{n}^{-1}(\mathbf{k})=1-\left.\partial_{\omega} \operatorname{Re} \Sigma_{n}(\mathbf{k}, \omega)\right|_{\omega=\varepsilon_{n}(\mathbf{k})}$, where $\Sigma_{n}(\mathbf{k}, \omega)$ is the self-energy due to the e-ph coupling for an electron in the $n$-th Bloch band. Using the ordinary Rayleigh-Schrödinger perturbation theory, one obtains

$$
\Sigma_{n}(\mathbf{k}, \omega)=\frac{1}{N} \sum_{\mathbf{q}, \lambda} \frac{\left|\gamma_{n n}^{\lambda}(\mathbf{k}, \mathbf{q})\right|^{2}}{\omega-\omega_{\lambda}-\varepsilon_{n}(\mathbf{k}+\mathbf{q})+i 0^{+}},
$$

where the dispersion of the optical phonons is neglected in the denominator of the last expression. In particular, the Rayleigh-Schrödinger perturbation theory is known to describe the properties of coupled e-ph systems at $\mathbf{k}=0$ better than the self-consistent Born approximation. ${ }^{18}$

In what follows, we compute the quasiparticle spectral weight due to the e-ph interaction for an electron at the bottom $(\mathbf{k}=0)$ of the conduction $(n \rightarrow c)$ band. From Eq. (7), for this special case we obtain
TABLE I. Calculated inverse quasiparticle weights (electron mass renormalization) $Z_{c}^{1}(0)=m_{\mathrm{eff}} / m_{\mathrm{e}}^{*}$ for various antidot lattices $\{L, R\}$, based on the phonon spectra obtained using the $4 \mathrm{NNFC}$ and VFF methods, respectively.

\begin{tabular}{rlllll}
\hline \hline & \multicolumn{2}{c}{$Z_{c}^{1}(0) 4 \mathrm{NNFC}$} & & \multicolumn{2}{c}{$Z_{c}^{1}(0) \mathrm{VFF}$} \\
\cline { 2 - 2 } \cline { 5 - 6 }$L$ & $R=5$ & $R=7$ & & $R=5$ & $R=7$ \\
\hline 9 & 5.046 & & & 4.811 & \\
10 & 4.836 & & & 4.613 & \\
11 & 4.732 & & & 4.509 & \\
12 & 4.681 & 4.056 & & 4.452 & 4.030 \\
13 & 4.662 & 3.827 & & 4.450 & 3.808 \\
14 & 4.668 & 3.739 & & 4.452 & 3.725 \\
15 & 4.684 & 3.707 & & 4.459 & 3.699 \\
16 & 4.709 & 3.758 & & 4.475 & 3.701 \\
17 & 4.733 & 3.795 & & 4.494 & 3.756 \\
\hline \hline
\end{tabular}

$$
Z_{c}^{1}(0)=1+\frac{1}{N_{\mathbf{q}, \lambda}} \sum_{\left[\varepsilon_{c}(0)-\varepsilon_{c}(\mathbf{q})-\omega_{\lambda}\right]^{2}},
$$

a quantity that yields the ratio of the effective (in the presence of the e-ph interaction) and the bare band electron masses: $Z_{c}^{-1}(0)=m_{\mathrm{eff}} / m_{\mathrm{e}}^{*}$. Based on Eq. (8), with the aid of Eqs. (5) and (6), we evaluate $Z_{c}^{-1}(0)$ for the $\{L, 5\} \quad(9 \leq L$ $\leq 17)$ and $\{L, 7\}(12 \leq L \leq 17)$ families of lattices. These demanding numerical calculations are performed via parallelization on multiple processors.

The calculations show a rather strong phonon-induced renormalization (see Table I) compared to graphene, ${ }^{19}$ where $Z=0.93$ (at the Dirac points) or larger. ${ }^{20}$ Importantly, we find a very good agreement between the results obtained using the 4NNFC and VFF phonon spectra, with the effective electron masses $m_{\text {eff }}$ from 4NNFC being slightly larger in all the cases considered. As can be inferred from Table I, for fixed $L$ the renormalization is larger for the structures with smaller antidot diameters, which squares with intuition. Another interesting feature that we find is a nonmonotonic $L$ dependence of $m_{\text {eff }}$ for given $R$, with minima for $L=13$ in the $\{L, 5\}$ family and $L=15$ in the $\{L, 7\}$ family of antidot lattices.

Detailed analysis shows that the low-energy phonons (below $30 \mathrm{meV}$ ) contribute at most 20 percent of the overall spectral weight, while among the high-energy ones the largest contributions come from two narrow intervals, around 173 and $194 \mathrm{meV}$, respectively. These high-energy modes typically provide $75-80$ percent of the spectral weight and their salient feature is that they do not involve significant atomic displacements in the vicinity of the antidot edges.

The obtained strong mass renormalization $m_{\mathrm{eff}} / m_{\mathrm{e}}^{*}$ $=3.7-5$ suggests that the charge carriers in the system acquire polaronic character. Indeed, it is plausible to have polaronic charge carriers in a narrow-band system with a strong e-ph coupling - a common situation in organic semiconductors $;{ }^{15}$ compared to the latter, graphene antidot lattices have yet narrower conduction bands and lower dimensionality. Given that the system at hand-due to its size 
and complexity - is out of reach of the exact-diagonalization methods, we have utilized a perturbative approach. Thus the obtained results are not expected to hold quantitatively, but they should still be qualitatively valid. These results underscore the relevance of phonons in antidot lattices and show that transport in these systems, unlike in graphene, cannot be treated as purely ballistic; i.e., it ought to be modeled by taking into account the inelastic degrees of freedom.

It is appropriate to comment on the robustness of our results for realistic antidot lattices, which may contain hydrogen $(\mathrm{H})$-terminated edges. The $\mathrm{H}$-atoms couple only to the $\sigma$-orbitals, while all the bands close to the Fermi energy originate from the $\pi$-electron states. These atoms give rise to a small change of hopping integrals near an edge due to the ensuing geometrical relaxation, leading to a minor band-gap modification. ${ }^{9}$ Likewise, the influence of a handful of $\mathrm{H}$-atoms on the dynamics of realistic antidot lattices is also not expected to be drastic, since-as shown in the present work-the dominant phonon modes involve only very small atomic displacements in the vicinity of edges. Therefore, while related issues certainly merit further investigation, our results are expected to be largely unaffected in realistic lattices.

In summary, we have studied the influence of phonons on the electronic properties of graphene antidot lattices. We have computed the phonon spectra for representative antidot lattices and determined the quasiparticle spectral weight due to the electron-phonon interaction for an electron at the bottom of the conduction band. We have shown that the phononinduced renormalization in these narrow-band systems is much stronger than in graphene itself, providing an indication of the polaronic nature of charge carriers. Our study paves the way for future investigations of the effect of phonons on the electronic and transport properties of graphene-based superlattices.

We thank C. Bruder for useful discussions. V.M.S. acknowledges financial support from the Swiss NSF and the NCCR Nanoscience. *vladimir.stojanovic@unibas.ch

${ }^{1}$ For a review, see A. K. Geim and K. S. Novoselov, Nature Mater. 6, 183 (2007); M. I. Katsnelson, Mater. Today 10, 20 (2007); A. H. Castro Neto et al., Rev. Mod. Phys. 81, 109 (2009); A. K. Geim, Science 324, 1530 (2009).

${ }^{2}$ See, e.g., P. Avouris et al., Nat. Nanotechnol. 2, 605 (2007); A. Cresti et al., Nano Res. 1, 361 (2008).

${ }^{3}$ V. V. Cheianov and V. I. Fal'ko, Phys. Rev. B 74, 041403(R) (2006); M. I. Katsnelson et al., Nat. Phys. 2, 620 (2006).

${ }^{4}$ C.-H. Park et al., Nat. Phys. 4, 213 (2008); Y. P. Bliokh, V. Freilikher, S. Savelev, and F. Nori, Phys. Rev. B 79, 075123 (2009); N. Abedpour, A. Esmailpour, R. Asgari, and M. R. Tabar, ibid. 79, 165412 (2009); R. P. Tiwari and D. Stroud, ibid. 79, 205435 (2009); W. Liu, Z. F. Wang, Q. W. Shi, J. Yang, and F. Liu, ibid. 80, 233405 (2009).

${ }^{5}$ T. G. Pedersen, C. Flindt, J. Pedersen, N. A. Mortensen, A. P. Jauho, and K. Pedersen, Phys. Rev. Lett. 100, 136804 (2008); T. G. Pedersen, C. Flindt, J. Pedersen, A. P. Jauho, N. A. Mortensen, and K. Pedersen, Phys. Rev. B 77, 245431 (2008).

${ }^{6}$ N. Shima and H. Aoki, Phys. Rev. Lett. 71, 4389 (1993).

${ }^{7}$ M. D. Fischbein and M. Drndić, Appl. Phys. Lett. 93, 113107 (2008); X. Jia et al., Science 323, 1701 (2009).

${ }^{8}$ M. Vanević, V. M. Stojanović, and M. Kindermann, Phys. Rev. B 80, 045410 (2009).

${ }^{9}$ J. A. Fürst, T. G. Pedersen, M. Brandbyge, and A. P. Jauho, Phys. Rev. B 80, 115117 (2009); New J. Phys. 11, 095020 (2009).

${ }^{10}$ T. Shen et al., Appl. Phys. Lett. 93, 122102 (2008).

${ }^{11}$ See, for example, J. Zaanen and P. B. Littlewood, Phys. Rev. B 50, 7222 (1994); V. M. Stojanović and M. Vanević, ibid. 78, 214301 (2008).

${ }^{12}$ R. Saito, T. Takeya, T. Kimura, G. Dresselhaus, and M. S. Dresselhaus, Phys. Rev. B 57, 4145 (1998); O. Dubay and G.
Kresse, ibid. 67, 035401 (2003); M. Mohr, J. Maultzsch, E. Dobardzic, S. Reich, I. Milosevic, M. Damnjanovic, A. Bosak, M. Krisch, and C. Thomsen, ibid. 76, 035439 (2007); J.-A. Yan, W. Y. Ruan, and M. Y. Chou, ibid. 77, 125401 (2008); J. Zimmermann, P. Pavone, and G. Cuniberti, ibid. 78, 045410 (2008); V. K. Tewary and B. Yang, ibid. 79, 075442 (2009); V. Perebeinos and J. Tersoff, ibid. 79, 241409(R) (2009); S. Viola Kusminskiy, D. K. Campbell, and A. H. Castro Neto, ibid. 80, 035401 (2009).

${ }^{13}$ W. P. Su, J. R. Schrieffer, and A. J. Heeger, Phys. Rev. Lett. 42, 1698 (1979).

${ }^{14}$ L. M. Woods and G. D. Mahan, Phys. Rev. B 61, 10651 (2000); G. D. Mahan, ibid. 68, 125409 (2003).

${ }^{15}$ See, e.g., K. Hannewald, V. M. Stojanović, J. M. T. Schellekens, P. A. Bobbert, G. Kresse, and J. Hafner, Phys. Rev. B 69, 075211 (2004); V. M. Stojanović, P. A. Bobbert, and M. A. J. Michels, ibid. 69, 144302 (2004).

${ }^{16}$ V. Perebeinos, J. Tersoff, and P. Avouris, Phys. Rev. Lett. 94, 086802 (2005); 94, 027402 (2005); L. E. F. Foa Torres and S. Roche, ibid. 97, 076804 (2006); Phys. Rev. B 75, 153402 (2007).

${ }^{17}$ L. D. Landau and E. M. Lifshitz, Statistical Mechanics, 6th ed., Course of Theoretical Physics (W. A. Benjamin, Reading, MA, 1979), Vol. V.

${ }^{18}$ G. D. Mahan, Many-Particle Physics (Plenum Press, New York, 1990).

${ }^{19}$ J. L. Mañes, Phys. Rev. B 76, 045430 (2007); M. Calandra and F. Mauri, ibid. 76, 205411 (2007); T. Stauber, N. M. R. Peres, and A. H. Castro Neto, ibid. 78, 085418 (2008); T. Stauber and N. M. R. Peres, J. Phys.: Condens. Matter 20, 055002 (2008).

${ }^{20}$ C.-H. Park, F. Giustino, M. L. Cohen, and S. G. Louie, Phys. Rev. Lett. 99, 086804 (2007). 